PROCEEDINGS OF THE

AMERICAN MATHEMATICAL SOCIETY

Volume 125, Number 7, July 1997, Pages 2093-2102

S 0002-9939(97)04003-3

\title{
JENSEN'S OPERATOR INEQUALITY FOR FUNCTIONS OF TWO VARIABLES
}

\author{
FRANK HANSEN
}

(Communicated by Palle E. T. Jorgensen)

\begin{abstract}
The operator convex functions of two variables are characterized in terms of a non-commutative generalization of Jensen's inequality.
\end{abstract}

\section{Functional CALCUlus FOR FunCtions OF SEVERAL VARIABlES}

The tensor product of two square matrices $A=\left(a_{i j}\right)$ and $B$ of order $n$ can be represented as the matrix

$$
A \otimes B=\left(\begin{array}{ccc}
a_{11} B & \cdots & a_{1 n} B \\
\vdots & & \vdots \\
a_{n 1} B & \cdots & a_{n n} B
\end{array}\right)
$$

which is of order $n^{2}$. However, if $A$ and $B$ are block matrices

$$
A=\left(\begin{array}{ll}
A_{11} & A_{12} \\
A_{21} & A_{22}
\end{array}\right), \quad B=\left(\begin{array}{ll}
B_{11} & B_{12} \\
B_{21} & B_{22}
\end{array}\right)
$$

of order $2 n$, then it is often more convenient to represent the tensor product $A \otimes B$ as the block matrix

$$
\left(\begin{array}{cccc}
A_{11} \otimes B_{11} & A_{11} \otimes B_{12} & A_{12} \otimes B_{11} & A_{12} \otimes B_{12} \\
A_{11} \otimes B_{21} & A_{11} \otimes B_{22} & A_{12} \otimes B_{21} & A_{12} \otimes B_{22} \\
A_{21} \otimes B_{11} & A_{21} \otimes B_{12} & A_{22} \otimes B_{11} & A_{22} \otimes B_{12} \\
A_{21} \otimes B_{21} & A_{21} \otimes B_{22} & A_{22} \otimes B_{21} & A_{22} \otimes B_{22}
\end{array}\right) .
$$

The definition according to (2) is unitarily equivalent to the definition according to (1), and no confusion will occur as long as the two representations are not mixed. The latter representation has the benefit of rendering formulas for block matrices more transparent and will be used throughout this paper. A similar representation will be used for tensor products of block matrices of bounded linear operators on a Hilbert space.

Korányi [10] considered functional calculus for functions of two variables. Let $f: I \times J \rightarrow \mathbf{R}$ be a function of two variables defined on the product of two intervals, and let $A, B$ be selfadjoint linear operators with finite spectra on a Hilbert space. If the spectrum of $A$ is contained in $I$, and the spectrum of $B$ is contained in $J$,

Received by the editors February 2, 1996.

1991 Mathematics Subject Classification. Primary 47A63; Secondary 47A80, 47Bxx.

(C)1997 American Mathematical Society 
and $A=\sum \lambda_{i} P_{i}$ and $B=\sum \mu_{j} Q_{j}$ are the spectral decompositions of $A$ and $B$ respectively, then the formula

$$
f(A, B)=\sum_{i, j} f\left(\lambda_{i}, \mu_{j}\right) P_{i} \otimes Q_{j}
$$

defines the functional calculus. The definition is readily extended to bounded normal operators and to functions of more than two variables. When we use the representation of tensor products of block matrices, then

$$
\begin{aligned}
f\left[\left(\begin{array}{cc}
A_{1} & 0 \\
0 & A_{2}
\end{array}\right),\left(\begin{array}{cc}
B_{1} & 0 \\
0 & B_{2}
\end{array}\right)\right] \\
\quad=\left(\begin{array}{cccc}
f\left(A_{1}, B_{1}\right) & 0 & 0 & 0 \\
0 & f\left(A_{1}, B_{2}\right) & 0 & 0 \\
0 & 0 & f\left(A_{2}, B_{1}\right) & 0 \\
0 & 0 & 0 & f\left(A_{2}, B_{2}\right)
\end{array}\right) .
\end{aligned}
$$

If the function $f$ can be separated as the product $f(t, s)=g(t) h(s)$ of two functions each of only one variable, then $f(A, B)=g(A) \otimes h(B)$.

The operator monotone functions of one variable were characterized by Löwner [12]. After a very important preparation by Kraus [11], this enabled Bendat and Sherman [4] to characterize the operator convex functions of one variable. There is no natural order structure for sets of operators, so the question of operator monotonicity for functions of several variables is not so fundamental. Korányi [10] nevertheless explored notions of operator monotonicity for functions of several variables and succeeded in characterizing such classes of functions. Work along this line has been continued by Vasudeva [13]. A function $f$ of $n$ real variables defined on a product $J=J_{1} \times \cdots \times J_{n}$ of intervals is said to be operator convex, if

$$
\begin{aligned}
& f\left(\lambda A_{1}+(1-\lambda) B_{1}, \ldots, \lambda A_{n}+(1-\lambda) B_{n}\right) \\
& \quad \leq \lambda f\left(A_{1}, \ldots, A_{n}\right)+(1-\lambda) f\left(B_{1}, \ldots, B_{n}\right)
\end{aligned}
$$

for every $\lambda \in[0,1]$ and all sequences of operators $\left(A_{1}, \ldots, A_{n}\right)$ and $\left(B_{1}, \ldots, B_{n}\right)$ such that the spectra of $A_{i}$ and $B_{i}$ are contained in $J_{i}$ for $i=1, \ldots, n$. This definition is meaningful since also the spectrum of $\lambda A_{i}+(1-\lambda) B_{i}$ is contained in the interval $J_{i}$ for $i=1, \ldots, n$. If $J$ is open, then it is enough to assume that $f$ is mid-point matrix convex for matrices of arbitrary order. This is so because a midpoint matrix convex function of order $4 n$ of one variable is automatically matrix convex of order $n$, cf. [7, The proof of Theorem 2.1]. The function $f$ is therefore real analytic in each variable and hence real analytic in $n$ variables according to Hartogs' theorem [8]. It is also clear that the pointwise limit of operator convex functions of $n$ variables is operator convex. Some operator convex functions of two variables have been studied by Ando [2] and Aujla [3] who also characterized the separately operator convex functions of two variables in terms of an operator inequality. In the case of one variable, it is well known that a continuous function $f:[0, \alpha[\rightarrow \mathbf{R}$ is operator convex (and $f(0) \leq 0$ ), if and only if it satisfies the inequality

$$
f\left(a^{*} x a\right) \leq a^{*} f(x) a
$$

for each selfadjoint operator $x$ with spectrum in $[0, \alpha[$ and each contraction $a$. This inequality can therefore be considered as a generalization of Jensen's inequality to operators [7]. It is the aim of this paper to generalize Jensen's operator inequality (JO) for functions of one variable to functions of two variables in such a way that it 
captures the notion of operator convexity. The proposed method will in principle be applicable to functions of $n$ variables, but the complexity is such that only functions of two variables are seriously considered. We obtain an operator inequality that characterizes the set of (normalized) operator convex functions of two variables, and we are able to characterize those separately operator convex functions of two variables that are (jointly) operator convex in terms of an operator inequality.

\section{UNITARY DILATIONS}

Theorem 2.1. Let $(a, b)$ be a pair of linear operators on a Hilbert space $H$ such that $a a^{*}+b b^{*}=1$ and $b$ is normal. There exists a normal operator $c$ such that

$$
U=\left(\begin{array}{cc}
a & b \\
c^{*} & -a^{*}
\end{array}\right)
$$

is unitary.

Proof. Let $\xi$ be a vector in $H$. Since $b$ is normal, we have

$$
\begin{aligned}
\left\|a^{*} b \xi\right\|^{2}=\left(a^{*} b \xi \mid a^{*} b \xi\right) & =\left(b \xi \mid a a^{*} b \xi\right) \\
& =\left(\xi \mid\left(1-b b^{*}\right) b b^{*} \xi\right) \\
& =\left(a a^{*} \xi \mid\left(1-a a^{*}\right) \xi\right) .
\end{aligned}
$$

If $a^{*} \xi=0$, it follows that $a^{*} b \xi=0$. We can thus define a linear operator $c$ on the range $R\left(a^{*}\right)$ of $a^{*}$ by setting

$$
c a^{*} \xi=a^{*} b \xi \quad \forall \xi \in H .
$$

Since $\left\|c a^{*} \xi\right\|=\left\|a^{*} b \xi\right\| \leq\left\|\left(1-a^{*} a\right)^{1 / 2}\right\| \cdot\left\|a^{*} \xi\right\|$, it follows that $c$ can be extended first by continuity to the closure of $R\left(a^{*}\right)$, and then to all of $H$ by setting $c=$ $1-p+c p$ where $p$ is the orthogonal projection on the closure of $R\left(a^{*}\right)$. We notice that $c a^{*}=a^{*} b$, and since $b$ is normal

$$
\begin{aligned}
a c^{*} a^{*} & =b^{*} a a^{*}=b^{*}\left(1-b b^{*}\right)=\left(1-b b^{*}\right) b^{*} \\
& =a a^{*} b^{*} .
\end{aligned}
$$

Thus $\left(c^{*} a^{*} \xi \mid a^{*} \eta\right)=\left(a^{*} b^{*} \xi \mid a^{*} \eta\right)$ for all $\xi, \eta \in H$. Since both $p H$ and $(1-p) H$ are left invariant by $c$ (and consequently by $c^{*}$ ), it follows that $c^{*} a^{*}=a^{*} b^{*}$. Furthermore,

$$
c^{*} c a^{*}=c^{*} a^{*} b=a^{*} b^{*} b=a^{*} b b^{*}=a^{*}\left(1-a a^{*}\right)=\left(1-a^{*} a\right) a^{*}
$$

and also

$$
c c^{*} a^{*}=c a^{*} b^{*}=a^{*} b b^{*}=a^{*}\left(1-a a^{*}\right)=\left(1-a^{*} a\right) a^{*} .
$$

It follows that

$$
c^{*} c=c c^{*}=1-p+\left(1-a^{*} a\right) p=1-a^{*} a,
$$

so $c$ is normal. Finally, we calculate

$$
U U^{*}=\left(\begin{array}{cc}
a & b \\
c^{*} & -a^{*}
\end{array}\right)\left(\begin{array}{cc}
a^{*} & c \\
b^{*} & -a
\end{array}\right)=\left(\begin{array}{cc}
a a^{*}+b b^{*} & a c-b a \\
c^{*} a^{*}-a^{*} b^{*} & c^{*} c+a^{*} a
\end{array}\right)=\left(\begin{array}{cc}
1 & 0 \\
0 & 1
\end{array}\right)
$$


and, using once more that $b$ is normal

$$
U^{*} U=\left(\begin{array}{cc}
a^{*} & c \\
b^{*} & -a
\end{array}\right)\left(\begin{array}{cc}
a & b \\
c^{*} & -a^{*}
\end{array}\right)=\left(\begin{array}{cc}
a^{*} a+c c^{*} & a^{*} b-c a^{*} \\
b^{*} a-a c^{*} & b^{*} b+a a^{*}
\end{array}\right)=\left(\begin{array}{cc}
1 & 0 \\
0 & 1
\end{array}\right) .
$$

\section{QED}

Let $(a, b)$ be a pair of operators on a Hilbert space $H$ such that $a a^{*}+b b^{*}=1$ and $b$ is normal, and let

$$
U=\left(\begin{array}{cc}
a & b \\
c^{*} & -a^{*}
\end{array}\right)
$$

be the unitary dilation constructed in Theorem 2.1. We notice that also

$$
V=\left(\begin{array}{cc}
a & -b \\
c^{*} & a^{*}
\end{array}\right)
$$

is unitary and calculate that

$$
U^{*}\left(\begin{array}{cc}
x & 0 \\
0 & 0
\end{array}\right) U=\left(\begin{array}{cc}
a^{*} x a & a^{*} x b \\
b^{*} x a & b^{*} x b
\end{array}\right), \quad V^{*}\left(\begin{array}{cc}
x & 0 \\
0 & 0
\end{array}\right) V=\left(\begin{array}{cc}
a^{*} x a & -a^{*} x b \\
-b^{*} x a & b^{*} x b
\end{array}\right) .
$$

Since the tensor product

$$
U \otimes U=\left(\begin{array}{cccc}
a \otimes a & a \otimes b & b \otimes a & b \otimes b \\
a \otimes c^{*} & -a \otimes a^{*} & b \otimes c^{*} & -b \otimes a^{*} \\
c^{*} \otimes a & c^{*} \otimes b & -a^{*} \otimes a & -a^{*} \otimes b \\
c^{*} \otimes c^{*} & -c^{*} \otimes a^{*} & -a^{*} \otimes c^{*} & a^{*} \otimes a^{*}
\end{array}\right)
$$

and

$$
V \otimes V=\left(\begin{array}{cccc}
a \otimes a & -a \otimes b & -b \otimes a & b \otimes b \\
a \otimes c^{*} & a \otimes a^{*} & -b \otimes c^{*} & -b \otimes a^{*} \\
c^{*} \otimes a & -c^{*} \otimes b & a^{*} \otimes a & -a^{*} \otimes b \\
c^{*} \otimes c^{*} & c^{*} \otimes a^{*} & a^{*} \otimes c^{*} & a^{*} \otimes a^{*}
\end{array}\right)
$$

we obtain that

$$
\begin{gathered}
(U \otimes U)^{*}\left(\begin{array}{cccc}
X & 0 & 0 & 0 \\
0 & 0 & 0 & 0 \\
0 & 0 & 0 & 0 \\
0 & 0 & 0 & 0
\end{array}\right)(U \otimes U)= \\
\left(\begin{array}{cccc}
\left(a^{*} \otimes a^{*}\right) X(a \otimes a) & \left(a^{*} \otimes a^{*}\right) X(a \otimes b) & \left(a^{*} \otimes a^{*}\right) X(b \otimes a) & \left(a^{*} \otimes a^{*}\right) X(b \otimes b) \\
\left(a^{*} \otimes b^{*}\right) X(a \otimes a) & \left(a^{*} \otimes b^{*}\right) X(a \otimes b) & \left(a^{*} \otimes b^{*}\right) X(b \otimes a) & \left(a^{*} \otimes b^{*}\right) X(b \otimes b) \\
\left(b^{*} \otimes a^{*}\right) X(a \otimes a) & \left(b^{*} \otimes a^{*}\right) X(a \otimes b) & \left(b^{*} \otimes a^{*}\right) X(b \otimes a) & \left(b^{*} \otimes a^{*}\right) X(b \otimes b) \\
\left(b^{*} \otimes b^{*}\right) X(a \otimes a) & \left(b^{*} \otimes b^{*}\right) X(a \otimes b) & \left(b^{*} \otimes b^{*}\right) X(b \otimes a) & \left(b^{*} \otimes b^{*}\right) X(b \otimes b)
\end{array}\right)
\end{gathered}
$$


for each element $X$ in the tensor product $B(H) \otimes B(H)$. We furthermore obtain that

$$
\begin{gathered}
(V \otimes V)^{*}\left(\begin{array}{cccc}
X & 0 & 0 & 0 \\
0 & 0 & 0 & 0 \\
0 & 0 & 0 & 0 \\
0 & 0 & 0 & 0
\end{array}\right)(V \otimes V)= \\
\left(\begin{array}{rrrr}
\left(a^{*} \otimes a^{*}\right) X(a \otimes a) & -\left(a^{*} \otimes a^{*}\right) X(a \otimes b) & -\left(a^{*} \otimes a^{*}\right) X(b \otimes a) & \left(a^{*} \otimes a^{*}\right) X(b \otimes b) \\
-\left(a^{*} \otimes b^{*}\right) X(a \otimes a) & \left(a^{*} \otimes b^{*}\right) X(a \otimes b) & \left(a^{*} \otimes b^{*}\right) X(b \otimes a) & -\left(a^{*} \otimes b^{*}\right) X(b \otimes b) \\
-\left(b^{*} \otimes a^{*}\right) X(a \otimes a) & \left(b^{*} \otimes a^{*}\right) X(a \otimes b) & \left(b^{*} \otimes a^{*}\right) X(b \otimes a) & -\left(b^{*} \otimes a^{*}\right) X(b \otimes b) \\
\left(b^{*} \otimes b^{*}\right) X(a \otimes a) & -\left(b^{*} \otimes b^{*}\right) X(a \otimes b) & -\left(b^{*} \otimes b^{*}\right) X(b \otimes a) & \left(b^{*} \otimes b^{*}\right) X(b \otimes b)
\end{array}\right)
\end{gathered}
$$

and consequently

$$
\begin{aligned}
& \frac{1}{2}(U \otimes U)^{*}\left(\begin{array}{cccc}
X & 0 & 0 & 0 \\
0 & 0 & 0 & 0 \\
0 & 0 & 0 & 0 \\
0 & 0 & 0 & 0
\end{array}\right)(U \otimes U)+\frac{1}{2}(V \otimes V)^{*}\left(\begin{array}{cccc}
X & 0 & 0 & 0 \\
0 & 0 & 0 & 0 \\
0 & 0 & 0 & 0 \\
0 & 0 & 0 & 0
\end{array}\right)(V \otimes V)= \\
& \left(\begin{array}{llll}
\left(a^{*} \otimes a^{*}\right) X(a \otimes a) & 0 & 0 & \left(a^{*} \otimes a^{*}\right) X(b \otimes b) \\
0 & \left(a^{*} \otimes b^{*}\right) X(a \otimes b) & \left(a^{*} \otimes b^{*}\right) X(b \otimes a) & 0 \\
0 & \left(b^{*} \otimes a^{*}\right) X(a \otimes b) & \left(b^{*} \otimes a^{*}\right) X(b \otimes a) & 0 \\
\left(b^{*} \otimes b^{*}\right) X(a \otimes a) & 0 & 0 & \left(b^{*} \otimes b^{*}\right) X(b \otimes b)
\end{array}\right) .
\end{aligned}
$$

A similar construction can be carried out on the tensor product $B(H) \otimes \cdots \otimes B(H)$ of $n$ copies of $B(H)$ in terms of $U \otimes \cdots \otimes U$ and $V \otimes \cdots \otimes V$.

\section{JENSEN'S OPERATOR INEQUALITY}

Theorem 3.1. If $f$ is a real continuous function of two variables defined on the domain $[0, \alpha[\times[0, \beta[$ (with $\alpha, \beta \leq \infty)$, the following conditions are equivalent:

(1) $f$ is operator convex, and $f(t, 0) \leq 0$ and $f(0, s) \leq 0$ for all $(t, s) \in$ $[0, \alpha[\times[0, \beta[$.

(2) The operator inequality

$$
\left(\begin{array}{cc}
f\left(a^{*} x a, a^{*} y a\right) & 0 \\
0 & f\left(b^{*} x b, b^{*} y b\right)
\end{array}\right) \leq\left(\begin{array}{cc}
(a \otimes a)^{*} f(x, y)(a \otimes a) & (a \otimes a)^{*} f(x, y)(b \otimes b) \\
(b \otimes b)^{*} f(x, y)(a \otimes a) & (b \otimes b)^{*} f(x, y)(b \otimes b)
\end{array}\right)
$$

is valid for all selfadjoint operators $x$ and $y$ with spectra in $[0, \alpha[$ and $[0, \beta[$ respectively, and all pairs of operators $(a, b)$ such that $a a^{*}+b b^{*}=1$ and $b$ is normal.

(3) The operator inequality

$$
\begin{aligned}
& \left(\begin{array}{cc}
f(p x p, p y p) & 0 \\
0 & f((1-p) x(1-p),(1-p) y(1-p))
\end{array}\right) \\
\leq & \left(\begin{array}{ll}
(p \otimes p) f(x, y)(p \otimes p) & (p \otimes p) f(x, y)((1-p) \otimes(1-p)) \\
((1-p) \otimes(1-p)) f(x, y)(p \otimes p) & ((1-p) \otimes(1-p)) f(x, y)((1-p) \otimes(1-p))
\end{array}\right)
\end{aligned}
$$


is valid for all selfadjoint operators $x$ and $y$ with spectra in $[0, \alpha[$ and $[0, \beta[$ respectively, and every orthogonal projection $p$.

Proof. $(1) \Rightarrow(2)$ :

Let $(a, b)$ be a pair of operators such that $a a^{*}+b b^{*}=1$ and $b$ is normal, and let $U$ and $V$ be the unitary dilations constructed in section 2 . Since $f$ is operator convex we obtain

$$
\begin{aligned}
& \left(\begin{array}{cccc}
f\left(a^{*} x a, a^{*} y a\right) & 0 & 0 & 0 \\
0 & f\left(a^{*} x a, b^{*} y b\right) & 0 & 0 \\
0 & 0 & f\left(b^{*} x b, a^{*} y a\right) & 0 \\
0 & 0 & 0 & f\left(b^{*} x b, b^{*} y b\right)
\end{array}\right) \\
& =f\left(\left(\begin{array}{cc}
a^{*} x a & 0 \\
0 & b^{*} x b
\end{array}\right),\left(\begin{array}{cc}
a^{*} y a & 0 \\
0 & b^{*} y b
\end{array}\right)\right) \\
& =f\left(\frac{1}{2} U^{*}\left(\begin{array}{cc}
x & 0 \\
0 & 0
\end{array}\right) U+\frac{1}{2} V^{*}\left(\begin{array}{cc}
x & 0 \\
0 & 0
\end{array}\right) V, \frac{1}{2} U^{*}\left(\begin{array}{ll}
y & 0 \\
0 & 0
\end{array}\right) U+\frac{1}{2} V^{*}\left(\begin{array}{cc}
y & 0 \\
0 & 0
\end{array}\right) V\right) \\
& \leq \frac{1}{2} f\left(U^{*}\left(\begin{array}{cc}
x & 0 \\
0 & 0
\end{array}\right) U, U^{*}\left(\begin{array}{cc}
y & 0 \\
0 & 0
\end{array}\right) U\right)+\frac{1}{2} f\left(V^{*}\left(\begin{array}{cc}
x & 0 \\
0 & 0
\end{array}\right) V, V^{*}\left(\begin{array}{cc}
y & 0 \\
0 & 0
\end{array}\right) V\right) \\
& =\frac{1}{2}(U \otimes U)^{*} f\left(\left(\begin{array}{cc}
x & 0 \\
0 & 0
\end{array}\right),\left(\begin{array}{cc}
y & 0 \\
0 & 0
\end{array}\right)\right)(U \otimes U) \\
& +\frac{1}{2}(V \otimes V)^{*} f\left(\left(\begin{array}{cc}
x & 0 \\
0 & 0
\end{array}\right),\left(\begin{array}{ll}
y & 0 \\
0 & 0
\end{array}\right)\right)(V \otimes V) \\
& =\frac{1}{2}(U \otimes U)^{*}\left(\begin{array}{cccc}
f(x, y) & 0 & 0 & 0 \\
0 & f(x, 0) & 0 & 0 \\
0 & 0 & f(0, y) & 0 \\
0 & 0 & 0 & f(0,0)
\end{array}\right)(U \otimes U) \\
& +\frac{1}{2}(V \otimes V)^{*}\left(\begin{array}{cccc}
f(x, y) & 0 & 0 & 0 \\
0 & f(x, 0) & 0 & 0 \\
0 & 0 & f(0, y) & 0 \\
0 & 0 & 0 & f(0,0)
\end{array}\right)(V \otimes V) \\
& \leq \frac{1}{2}(U \otimes U)^{*}\left(\begin{array}{cccc}
f(x, y) & 0 & 0 & 0 \\
0 & 0 & 0 & 0 \\
0 & 0 & 0 & 0 \\
0 & 0 & 0 & 0
\end{array}\right)(U \otimes U) \\
& +\frac{1}{2}(V \otimes V)^{*}\left(\begin{array}{cccc}
f(x, y) & 0 & 0 & 0 \\
0 & 0 & 0 & 0 \\
0 & 0 & 0 & 0 \\
0 & 0 & 0 & 0
\end{array}\right)(V \otimes V)
\end{aligned}
$$




$$
=\left(\begin{array}{ll}
(a \otimes a)^{*} f(x, y)(a \otimes a) & 0 \\
0 & (a \otimes b)^{*} f(x, y)(a \otimes b) \\
0 & (b \otimes a)^{*} f(x, y)(a \otimes b) \\
(b \otimes b)^{*} f(x, y)(a \otimes a) & 0
\end{array}\right.
$$

$$
\left.\begin{array}{ll}
0 & (a \otimes a)^{*} f(x, y)(b \otimes b) \\
(a \otimes b)^{*} f(x, y)(b \otimes a) & 0 \\
(b \otimes a)^{*} f(x, y)(b \otimes a) & 0 \\
0 & (b \otimes b)^{*} f(x, y)(b \otimes b)
\end{array}\right) .
$$

In particular,

$$
\left(\begin{array}{cc}
f\left(a^{*} x a, a^{*} y a\right) & 0 \\
0 & f\left(b^{*} x b, b^{*} y b\right)
\end{array}\right) \leq\left(\begin{array}{cc}
(a \otimes a)^{*} f(x, y)(a \otimes a) & (a \otimes a)^{*} f(x, y)(b \otimes b) \\
(b \otimes b)^{*} f(x, y)(a \otimes a) & (b \otimes b)^{*} f(x, y)(b \otimes b)
\end{array}\right) .
$$

$(2) \Rightarrow(3)$ is obvious.

$(3) \Rightarrow(1)$ : Let $\left(x_{1}, x_{2}\right)$ and $\left(y_{1}, y_{2}\right)$ be pairs of operators with spectra in $[0, \alpha[$ and $[0, \beta[$ respectively. We set

$$
U=\frac{\sqrt{2}}{2}\left(\begin{array}{cc}
1 & -1 \\
1 & 1
\end{array}\right)
$$

and

$$
x=U^{*}\left(\begin{array}{cc}
x_{1} & 0 \\
0 & x_{2}
\end{array}\right) U, \quad y=U^{*}\left(\begin{array}{cc}
y_{1} & 0 \\
0 & y_{2}
\end{array}\right) U, \quad p=\left(\begin{array}{ll}
1 & 0 \\
0 & 0
\end{array}\right) .
$$

It follows from (3) that

$$
\begin{aligned}
& \left(\begin{array}{cc}
f\left(\left(\begin{array}{cc}
\frac{x_{1}+x_{2}}{2} & 0 \\
0 & 0
\end{array}\right),\left(\begin{array}{cc}
\frac{y_{1}+y_{2}}{2} & 0 \\
0 & 0
\end{array}\right)\right) & f\left(\left(\begin{array}{cc}
0 & 0 \\
0 & \frac{x_{1}+x_{2}}{2}
\end{array}\right),\left(\begin{array}{cc}
0 & 0 \\
0 & \frac{y_{1}+y_{2}}{2}
\end{array}\right)\right)
\end{array}\right) \\
& =\left(\begin{array}{cc}
f(p x p, p y p) & 0 \\
0 & f((1-p) x(1-p),(1-p) y(1-p))
\end{array}\right) \\
& \leq\left(\begin{array}{cc}
(p \otimes p) f(x, y)(p \otimes p) & (p \otimes p) f(x, y)((1-p) \otimes(1-p)) \\
((1-p) \otimes(1-p)) f(x, y)(p \otimes p) & ((1-p) \otimes(1-p)) f(x, y)((1-p) \otimes(1-p))
\end{array}\right) .
\end{aligned}
$$

All rows and columns with indices from 2 to 7 in the last $8 \times 8$ block matrix contain only the zero operator on $H$. In particular,

$$
f\left(\frac{x_{1}+x_{2}}{2}, 0\right) \leq 0 \text { and } f\left(0, \frac{y_{1}+y_{2}}{2}\right) \leq 0
$$

and it follows that $f(t, 0) \leq 0$ and $f(0, s) \leq 0$ for all $(t, s) \in[0, \alpha[\times[0, \beta[$. Since

$$
U \otimes U=\frac{1}{2}\left(\begin{array}{cccc}
1 & -1 & -1 & 1 \\
1 & 1 & -1 & -1 \\
1 & -1 & 1 & -1 \\
1 & 1 & 1 & 1
\end{array}\right)
$$


we calculate the element in the first row and first column of the $4 \times 4$ block matrix

$$
\begin{aligned}
& (p \otimes p) f(x, y)(p \otimes p) \\
& \quad=(p \otimes p)(U \otimes U)^{*} f\left(\left(\begin{array}{cc}
x_{1} & 0 \\
0 & x_{2}
\end{array}\right),\left(\begin{array}{cc}
y_{1} & 0 \\
0 & y_{2}
\end{array}\right)\right)(U \otimes U)(p \otimes p)
\end{aligned}
$$

to be $\left(f\left(x_{1}, y_{1}\right)+f\left(x_{1}, y_{2}\right)+f\left(x_{2}, y_{1}\right)+f\left(x_{2}, y_{2}\right)\right) / 4$. This is also the entry in the fourth row and fourth column of the $4 \times 4$ block matrix $((1-p) \otimes(1-p)) f(x, y)$ $\cdot((1-p) \otimes(1-p))$ while the first row and fourth column of the $4 \times 4$ block matrix $(p \otimes p) f(x, y)((1-p) \otimes(1-p))$ and the fourth row and first column of the transpose both are $\left(f\left(x_{1}, y_{1}\right)-f\left(x_{1}, y_{2}\right)-f\left(x_{2}, y_{1}\right)+f\left(x_{2}, y_{2}\right)\right) / 4$. Inserting these values in the above inequality between $8 \times 8$ block matrices and then discarding the rows and columns with indices between 2 and 7 , it follows that

$$
\begin{aligned}
& f\left(\frac{x_{1}+x_{2}}{2}, \frac{y_{1}+y_{2}}{2}\right)\left(\begin{array}{ll}
1 & 0 \\
0 & 1
\end{array}\right) \\
\leq & \frac{f\left(x_{1}, y_{1}\right)+f\left(x_{2}, y_{2}\right)}{4}\left(\begin{array}{ll}
1 & 1 \\
1 & 1
\end{array}\right)+\frac{f\left(x_{1}, y_{2}\right)+f\left(x_{2}, y_{1}\right)}{4}\left(\begin{array}{cc}
1 & -1 \\
-1 & 1
\end{array}\right) .
\end{aligned}
$$

By multiplying the above operator inequality to the left and to the right with the projection $\frac{1}{2}\left(\begin{array}{ll}1 & 1 \\ 1 & 1\end{array}\right)$, we obtain

$$
\frac{1}{2} f\left(\frac{x_{1}+x_{2}}{2}, \frac{y_{1}+y_{2}}{2}\right)\left(\begin{array}{cc}
1 & 1 \\
1 & 1
\end{array}\right) \leq \frac{f\left(x_{1}, y_{1}\right)+f\left(x_{2}, y_{2}\right)}{4}\left(\begin{array}{cc}
1 & 1 \\
1 & 1
\end{array}\right)
$$

and consequently

$$
f\left(\frac{x_{1}+x_{2}}{2}, \frac{y_{1}+y_{2}}{2}\right) \leq \frac{1}{2} f\left(x_{1}, y_{1}\right)+\frac{1}{2} f\left(x_{2}, y_{2}\right) .
$$

Since $f$ is continuous, it follows that $f$ is operator convex. QED

Let $\sigma$ be the extremal connection associated with the representing function $f(t)=-t^{2}$, cf. [6, Theorem 2.6]. The mapping $(A, B) \rightarrow A \sigma B$ is concave on the set of pairs of positive semi-definite operators, and if $A$ is invertible given by the formula

$$
A \sigma B=A^{1 / 2} f\left(A^{-1 / 2} B A^{-1 / 2}\right) A^{1 / 2}=-B A^{-1} B .
$$

It is connected to the harmonic mean through the formula

$$
A ! B=2[B+(A+B) \sigma B] .
$$

We shall characterize the operator convex functions of two variables among the separately operator convex functions in terms of the connection $\sigma$. The following result can be deduced by slightly altering the presentation in Aujla [3].

Proposition 3.2. Let $f$ be a real continuous function of two variables defined on the domain $[0, \alpha[\times[0, \beta[($ with $\alpha, \beta \leq \infty)$ and suppose that $f(t, 0) \leq 0$ and $f(0, s) \leq$ 0 for all $(t, s) \in[0, \alpha[\times[0, \beta[$. Then $f$ is separately operator convex, if and only if the difference

$$
\Delta(a)=(a \otimes a)^{*} f(x, y)(a \otimes a)-f\left(a^{*} x a, a^{*} y a\right) \geq 0
$$

for all selfadjoint operators $x$ and $y$ with spectra in $[0, \alpha[$ and $[0, \beta[$ respectively, and every contraction a. 
Theorem 3.3. Let $f$ be a separately operator convex function of two variables defined on the domain $[0, \alpha[\times[0, \beta[$ (with $\alpha, \beta \leq \infty)$ and suppose that $f(t, 0) \leq 0$ and $f(0, s) \leq 0$ for all $(t, s) \in[0, \alpha[\times[0, \beta[$. Then $f$ is (jointly) operator convex, if and only if

$$
\left[V^{*} \Delta(a) V\right] \sigma|C|+\Delta(b) \geq 0
$$

where $C=V|C|$ is the polar decomposition of $C=(a \otimes a)^{*} f(x, y)(b \times b)$, for all selfadjoint operators $x$ and $y$ with spectra in $[0, \alpha[$ and $[0, \beta[$ respectively, and all pairs of operators $(a, b)$ such that $a a^{*}+b b^{*}=1$ and $b$ is normal. The condition remains sufficient if $a$ is constrained to be of the form $a=p$, where $p$ is an orthogonal projection, and $b=1-p$.

Proof. If $f$ is operator convex, then

$$
\left(\begin{array}{cc}
V^{*} \Delta(a) V & |C| \\
|C| & \Delta(b)
\end{array}\right)=\left(\begin{array}{cc}
V & 0 \\
0 & 1
\end{array}\right)^{*}\left(\begin{array}{cc}
\Delta(a) & C \\
C^{*} & \Delta(b)
\end{array}\right)\left(\begin{array}{cc}
V & 0 \\
0 & 1
\end{array}\right) \geq 0
$$

according to Theorem 3.1, and this is equivalent to

$$
\left[V^{*} \Delta(a) V\right] \sigma|C|+\Delta(b) \geq 0,
$$

cf. [2, Lemma 1] or [1]. Suppose conversely that

$$
\left[V^{*} \Delta(p) V\right] \sigma|C|+\Delta(1-p) \geq 0
$$

for each orthogonal projection $p$. The statement is equivalent to

$$
\left(\begin{array}{cc}
r(C) \Delta(p) r(C) & C \\
C^{*} & \Delta(1-p)
\end{array}\right) \geq 0
$$

where $r(C)$ is the range projection of $C=(p \otimes p) f(x, y)((1-p) \otimes(1-p))$. In particular, if we restrict ourselves to such projections that are considered in the proof of $3.1(3) \Rightarrow(1)$, it follows that $r(C)=p \otimes p$ whenever

$$
f\left(x_{1}, y_{1}\right)-f\left(x_{1}, y_{2}\right)-f\left(x_{2}, y_{1}\right)+f\left(x_{2}, y_{2}\right)
$$

is invertible. Since $(p \otimes p) \Delta(p)(p \otimes p) \leq \Delta(p)$ by virtue of the normalization of $f$, we obtain

$$
\left(\begin{array}{cc}
\Delta(p) & C \\
C^{*} & \Delta(1-p)
\end{array}\right) \geq 0
$$

and hence

$$
f\left(\frac{x_{1}+x_{2}}{2}, \frac{y_{1}+y_{2}}{2}\right) \leq \frac{1}{2} f\left(x_{1}, y_{1}\right)+\frac{1}{2} f\left(x_{2}, y_{2}\right)
$$

whenever the expression in $(*)$ is invertible. If the function $f$ is constant in one of the variables, then $f$ is trivially operator convex. Otherwise we obtain that the expression in $(*)$ is invertible and thus the inequality $(\#)$ is satisfied for $x_{1}, x_{2}, y_{1}, y_{2}$ belonging to a dense set of operators. Since $f$ is continuous, it follows that $f$ is operator convex. QED 


\section{REFERENCES}

1. W.N. Anderson, Jr., Shorted operators, SIAM J. Appl. Math., 20:520-525, 1971. MR 44:5172

2. T. Ando, Concavity of certain maps of positive definite matrices and applications to Hadamard products, Linear Algebra Appl., 26:203-241, 1979. MR 80f:15023

3. J.S. Aujla, Matrix convexity of functions of two variables, Linear Algebra and Its Applications, 194:149-160, 1993.

4. J. Bendat and S. Sherman, Monotone and convex operator functions, Trans. Am. Math. Soc., 79:58-71, 1955. MR 18:588b

5. F. Hansen, An operator inequality, Math. Ann., 246:249-250, 1980. MR 82a:46065

6. F. Hansen, Extrema for concave operator mappings, Math. Japonica., 40:331-338, 1994. MR 95h: 47019

7. F. Hansen and G.K. Pedersen, Jensen's inequality for operators and Löwner's theorem, Math. Ann., 258:229-241, 1982. MR 83g:47020

8. F. Hartogs, Zur Theorie der analytischen Funktionen mehrerer unabhängiger Veränderlichen, inbesonderere über die Darstellung derselben durch Reihen, welche nach Potenzen einer Veränderlichen fortschreiten, Math. Ann., 62:1-88, 1906.

9. R.V. Kadison and J.R. Ringrose, Fundamentals of the Theory of Operator Algebras, I-II, Academic Press, 1982 \& 1986.

10. A. Korányi, On some classes of analytic functions of several variables, Trans Amer. Math. Soc., 101:520-554, 1961. MR 25:226

11. F. Kraus, Über konvekse Matrixfunktionen, Math. Z., 41:18-42, 1936.

12. K. Löwner, Über monotone Matrixfunktionen, Math. Z., 38:177-216, 1934.

13. H. Vasudeva, On monotone matrix functions of two variables, Trans. Amer. Math. Soc., 176:305-318, 1973. MR 47:2408

Institute of Economics, University of Copenhagen, Studiestraede 6, DK-1455 CopenHAGEN K, DENMARK 